\title{
The Implicit Aggression of Indirect Bullies and Victims in Chinese Universities in Memory-guided Saccades
}

\author{
Zhongling Pi, Lirong Xia, Sheng Xu, Yutang Li, Chuang Gao, Jianzhong Hong \\ Key Laboratory of Adolescent Cyberpsychology and Behavior (CCNU) \\ Ministry of Education School of Psychology \\ Central China Normal University \\ Wuhan 430079, Hubei Province, China
}

\begin{abstract}
This study examined the implicit aggression of Chinese undergraduates who were involved and not involved in indirect bullying. The participants consisted of 36 Chinese undergraduates, and the revised version of Olweus Bully/Victim Questionnaire for Chinese was adopted. Indirect bullying situational pictures and responding situational pictures to the pictures (aggression and non-aggression) were used to investigate undergraduates' automatic responses in the memory-guided saccade paradigm based on eye movement technology. The results showed that indirect bullies preferred to aggressive responses. It indicated that indirect bullies were more implicitly aggressive than victims and undergraduates who were not involved. The paper suggests that indirect bullies should be informed how to release their implicit aggression appropriately.
\end{abstract}

Keywords-undergraduate; Chinese university; indirect bully; indirect victim; implicit aggression; eye-movement; memory-guided saccade paradigm

\section{INTRODUCTION}

Bullying behaviors threaten the security of society and increase the rate of social crimes. They receive attention from many researchers in social sciences. Bullying is a negative and repeated incident between the same individuals over a prolonged period of time, with the intention to harm the victim. It implies the power imbalance between the victim and the bully [1-2]. Bullying can be direct and indirect. The indirect bullying includes relational harassment and spreading rumors by social rejection or social exclusion [3]. It is more insidious. Indirect bullying has many negative consequences on psychological development. It is associated with a significant reduction in self-esteem, and increases involvement in emotional problems, delinquent behavior and drug use. Moreover, those who experience indirect bullying are more likely to report membership in a gang [4-5].

Indirect bullying is relatively common in universities. $\mathrm{Li}$ (2010), for example, found that $7.33 \%$ of 941 undergraduates bully others indirectly, and $8.61 \%$ were bullied indirectly by others. Results of the study showed that indirect bullying was more prevalent as compared with direct bullying in universities [6]. In China, indirect bullying is very common among undergraduates in universities, and our media are very much concerned with it and have reported it for many times [7]. However, it has so far not yet been received much attention by academics. Thus, the present study was designed to explore the indirect bullying in Chinese universities.

Previous research on indirect bullying in universities focused mainly on explicit aggressive behaviors [8-9], but little was known about the implicit aggression of bullies and victims, which was vital to explore the reason why indirect bullies bullied others, and serve as an important guide for those engaged in intervention with it. For implicit aggression, researchers generally measured it through the Implicit Association Test (IAT). However, there were some controversies on IAT. For example, some researchers argued that IAT test assessed familiarity [10], others assumed that it assessed perceptual salience asymmetries [11], or mere cultural knowledge irrespective of personal endorsement of that knowledge [12]. In addition, researchers claimed that the results of IAT test might be affected by participants' cognitive capabilities, such as their capability to adjust to switching the first category to the second category [13]. The memory-guided saccade paradigm could be used to investigate preference to stimulus and would overcome the above-mentioned limitations. Thus, the goal of the study was to explore the implicit aggression of undergraduates who were involved and not involved in indirect bullying in Chinese universities through memory-guided saccade paradigm.

This study used bullying situational pictures and responding situational pictures to the pictures (aggression and non-aggression) to investigate the implicit aggression of indirect bullies, victims and undergraduates who were not involved. According to previous research, it was found that implicit aggression could serve as reliable predictors of individual's explicit aggressive behavior [14]. In fact, bullying is a special type of aggressive behavior. Therefore, our hypothesis was that compare with indirect victims and undergraduates not involved, indirect bullies are more implicitly aggressive.

\section{METHODS}

\section{A. Participants}

The participants were 500 Chinese undergraduates at the beginning of the study ( 288 girls and 212 boys, 95 freshmen, 168 sophomores, 152 juniors, and 85 seniors), with a mean age of 20.3 years ( $S D=2.6$ years), from seven universities in Wuhan, China. All participants were administered the 
revised version of Olweus Bully/Victim Questionnaire (for further information in Appendix 1). The questionnaire detected and determined participants' roles (indirect bullies, victims and individuals who were not involved) in indirect bullying.

Ultimately, the suitable participant selected for the following experiment consisted of 36 undergraduates (17 boys and 19 girls; $\mathrm{M}=21.4$ years, $\mathrm{SD}=3.4$ years). Of these, 12 undergraduates were indirect bullies, 12 indirect victims and 12 undergraduates not involved. All participants had not participated in eye movement experiment before and had normal or corrected-to-normal vision. They all gave written informed consent.

\section{B. Stimuli}

The original stimuli were two bullying photographs (see Figure 1), and 16 responding situational photographs (8 were for boys and 8 for girls). In these responding situational photographs, 6 were aggressive situations (4 for direct aggressive situations, 2 for indirect aggressive situations), and 2 were non-aggressive situations (1 for staying alone situation and 1 for having a good time together). All photographs' backgrounds were removed by Photoshop cs5. All photographs were in black and white. A bullying picture in the center and 8 responding pictures were combined into a picture, called the combined picture. They were same gender and were $576 \mathrm{~cm}$ in height and $768 \mathrm{~cm}$ in width (see Figure 2, a \& b). In order to avoid position effect on the undergraduates' automatic responses, each responding situational picture was presented at all positions. In this way, there were 64 combined pictures.
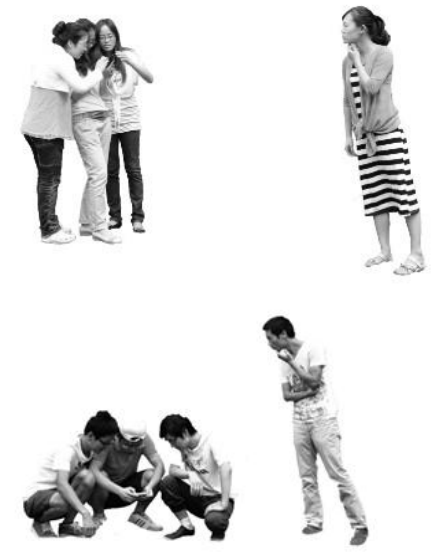

Figure 1. Indirect bullying situational pictures
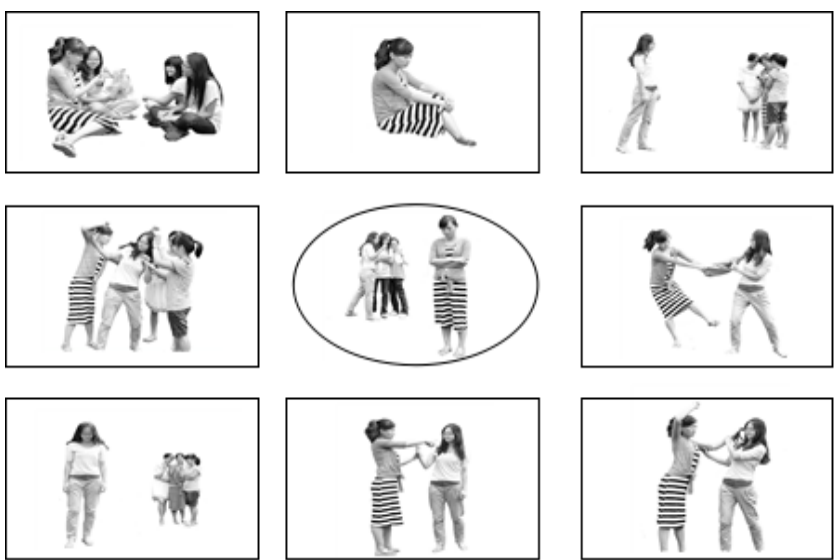

a
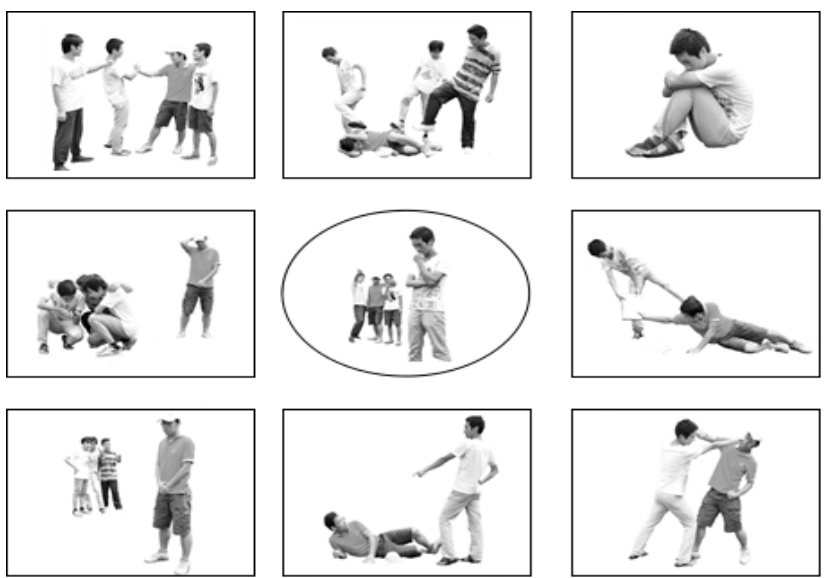

b

Figure 2. The combined pictures

\section{Apparatus}

The stimuli were presented on a 21 -in. CRT monitor (NESOJXC FS210A) at a viewing distance of $80 \mathrm{~cm}$. The resolution of the monitor was $1024 \times 768$ pixels and the refresh rate was $75 \mathrm{~Hz}$. Participants used a chin rest with a head-stop to stabilize head position during experiment. Eye movements were registered by Eyelink 1000 (SR Research Ltd., Mississauga, Ontario, Canada) non-invasive infrared eye tracker. The Eyelink 1000 Tower Mount system had a $1000 \mathrm{~Hz}$ binocular temporal resolution and $2000 \mathrm{~Hz}$ monocular sampling rate and a $<0.01^{\circ}$ of gaze resolution and a gaze position accuracy of $<0.5^{\circ}$. The manufacturer's software was used for calibration, validation, determining periods of fixation and stimuli presentation. Data from binocular was monitored.

\section{Task}

Some details of the classical paradigm were changed according to the purpose of this experiment. In a typical trial, participants were first presented with a bullying situational picture in the center of screen for $2000 \mathrm{~ms}$, then, they were presented with 8 responding situational pictures for $1000 \mathrm{~ms}$. After these responding situational pictures 
disappearing, the bullying picture and, an arrow, as imperative cue, were presented and the arrow points a position of responding situational pictures for $50 \mathrm{~ms}$. The direction of arrow was random. Then the bullying picture was presented alone for $1500 \mathrm{~ms}$.

\section{E. Procedure}

The experiment was conducted in a dimly lit room. The experiment consisted of two stages. Before the start of experiment start, the participants were informed about the concept of bully and classification. The first stage was calibration. Participants had to fixate nine calibration targets that were present randomly in a $3 \times 3$ grid across the monitor. Participants were instructed to fixate the center fixation point and the fixation point changed into a plus sign as an indication that the positions of the eyes were recalibrated. After the recalibration, the formal experiment started. There were 64 trials. There was an empty screen between two trials for $1000 \mathrm{~ms}$. The total experiment lasted 15 minutes.
The actual introductions were as follows:' after the start of experiment, you will see an indirect bullying picture on the screen, and then you will see 8 situations of responses. You should fix the bullying picture all the time until an arrow appears. At this point, you saccade to the response the arrow directing at as soon as possible.'

\section{RESULTS}

In order to assess the undergraduates' preference, we analyzed participants' saccadic movements and saccadic time to the situations of responses after arrow presented. These responses were divided into aggressive (indirect aggressive and direct aggressive) and non-aggressive.

\section{A. Preliminary Analysis}

The means and standard deviations of the saccadic time to the situations of responses of indirect bullies, victims and undergraduates not involved were shown in Table 1.

TABLE I. MEANS OF THE SACCADIC TIME TO THE SITUATIONS OF RESPONSES

\begin{tabular}{lllll}
\hline & Aggression & Direct aggression & Indirect aggression & Non-aggression \\
\hline $\begin{array}{l}\text { Indirect } \\
\text { bullies }\end{array}$ & $947.16(148.497)$ & $955.54(153.262)$ & $930.42(162.558)$ & $950.36(156.597)$ \\
\hline $\begin{array}{l}\text { Indirect } \\
\text { victims }\end{array}$ & $1071.75(125.690)$ & $1053.90(124.693)$ & $1107.45(148.693)$ & $1053.84(154.800)$ \\
\hline $\begin{array}{l}\text { Not } \\
\text { involved }\end{array}$ & $1040.94(82.433)$ & $1034.27(80.000)$ & $1054.28(103.281)$ & $1075.25(102.156)$ \\
\hline
\end{tabular}

\section{B. Participant Roles and Implicit Aggression}

Firstly, we examined whether the time of saccade to aggressive responses or non-aggressive responses was different between indirect bullies, victims, and those not involved. A 3 (role) $\times 2$ (type of responses) repeated measures ANOVAs was conducted on the time of saccade to two types of situations of responses, with role as between factor. The first main effect of type of responses was not significant (Pillai's Trace $=0.012, \mathrm{~F}(1,33)=0.415, \mathrm{P}>$ $0.05)$. The second main effect of role reached a level of marginal significance $(\mathrm{F}(2,33)=3.058, \mathrm{P}=0.060)$. The third effect for the interaction between role and type of responses was not significant (Pillai's Trace $=0.119, \mathrm{~F}(2$, $33)=2.238, \mathrm{P}>0.05)$. Then two separate One-Way ANOVAs were conducted on the mean duration time of saccade to response (aggressive responses or non-aggressive responses), with role as independent factor. There was no significant difference in non-aggressive responses among indirect bullies, victims and those not involved $(\mathrm{F}(2,33)=2.725, \mathrm{P}>0.05)$. But there was significant difference in aggressive responses among three groups $(\mathrm{F}(2,33)=3.395, \mathrm{P}<0.05)$. Post hoc analyses revealed that the indirect bullies' time saccade to aggressive responses was significant shorter than victims' (LSD comparisons).

To further analysis, the aggressive responses were divided into direct aggressive responses and indirect aggressive responses. A 3 (role) $\times 3$ (type of responses) repeated measures ANOVAs was conducted on the time of saccade to two types of situations of responses (direct aggressive responses, indirect aggressive responses, and non-aggressive responses), with role as between factor. The first main effect of type of responses was not significant (Pillai's Trace $=0.047, \mathrm{~F}(2,32)=0.752, \mathrm{P}>0.05)$. The second main effect of role was significant $(F(2,33)=3.534$, $\mathrm{P}<0.05)$. The third effect for the interaction between role and type of responses was significant (Pillai's Trace = $0.238, F(4,66)=2.402, \mathrm{P}<0.05$, see figure 3.). Simple effect analysis found out that no significant difference was in non-aggressive responses or direct aggressive responses among three groups $(F(2,33)=2.147, P>0.05 ; F(2,33)$ $=2.15, P>0.05)$. But there was significant difference in indirect bullying responses $(F(2,33)=5.02, P<0.05)$. Post hoc analyses revealed that the indirect bullies' time saccade to indirect bullying responses was significant shorter than victims' and those not involved (LSD comparisons). 


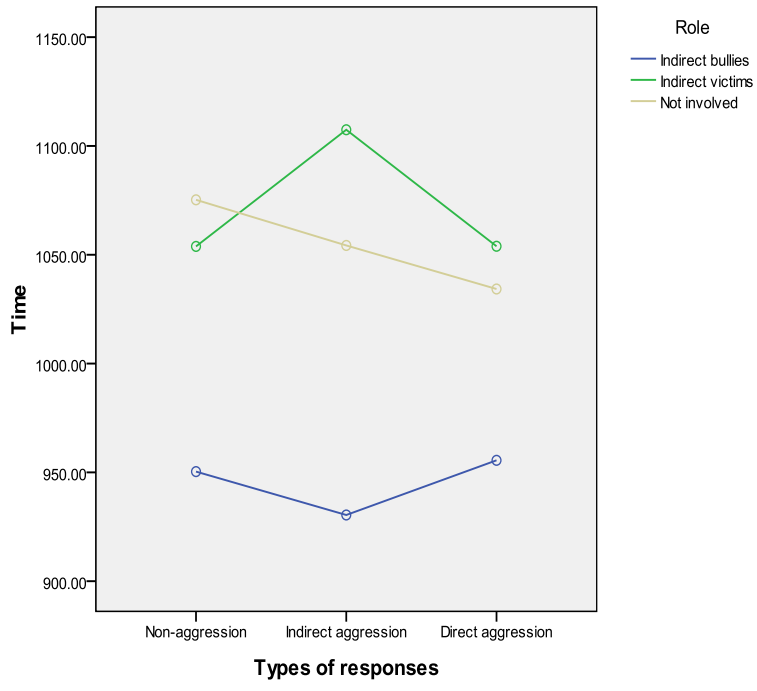

Figure 3. Interaction effect between role and type of responses

These results supported our hypotheses. It was found that compare with victims and undergraduates not involved, bullies selected aggressive responses automatically, in particular, indirect aggressive responses.

\section{DISCUSSIONS AND CONCLUSION}

In our study, we found out the indirect bullies preferred to indirectly aggressive responses, implying that they had more implicit aggression than that of victims and undergraduates not involved, which may be one of main reasons why they often bullied others. It was worth mentioning that we used the memory-guided saccade paradigm to explore the participants' implicit aggression. In this paradigm, the participants were instructed to suppress the reflexive eye movement, saccade to a response that they wanted to select, and fix on a center picture [15]. Then participants saccade to the response that an arrow directed at. If the participant was interested to one responding situation among the surrounding pictures, he would be able to saccade to that response quickly. Responding pictures were presented $1000 \mathrm{~ms}$. For this reason, it was no time for thinking over response, so that the saccade in this paradigm might reflect people's automatic response towards bullying situations. Therefore, this paradigm could be used in future research on implicit aggression.

There were several limitations in the study. The most prominent problem was that we only explored the implicit social cognitions of 12 indirect bullies, 12 indirect victims and 12 undergraduates not involved in Chinese universities. The sample size was small and the conclusion generalized to the whole undergraduate of the same and other schools should be cautious. Further research should enlarge sample size.

In sum, there were some interesting outcomes derived from our study. Indirect bullies were more implicitly aggressive than victims and undergraduates not involved. Therefore, we might assume that indirect bullies' implicit aggression could lead bullying, and the experiences of exposure to bully might have negative effects on their implicit social cognition. Our results provide an important guide for future indirect bullying intervention, that is, we should inform bullies how to release their implicit aggression appropriately. Bullies could participate in competition activities, such as wrestling, playing computer games and boxing.

\section{ACKNOWLEDGMENT}

Thank you to all the participants and co-authors. This research was financially supported by Social Science Foundation of The Chinese Education Commission grant 15609540 .

\section{REFERENCES}

[1] Olweus, D. (1991). Bully/victim problems among schoolchildren: Basic facts and effects of a school based intervention program. The development and treatment of childhood aggression, 411-448.

[2] Olweus, D. (1993). Bullying at school: What we know and what we can do. Oxford, UK: Basil Blackwell.

[3] Rivers, I., \& Smith, P. K. (1994). Types of bullying behaviour and their correlates. Aggressive Behavior, 20(5), 359-368.

[4] Baldry, A. C. (2004). The impact of direct and indirect bullying on the mental and physical health of Italian youngsters. Aggressive Behavior, 30(5), 343-355.

[5] Carbone-Lopez, K., Esbensen, F. A., \& Brick, B. T. (2010). Correlates and consequences of peer victimization: Gender differences in direct and indirect forms of bullying. Youth Violence and Juvenile Justice, $8(4), 332-350$.

[6] Li, M. (2010). Research on bullying behavior of college students in Liaoning Province (in Chinese). Journal of Liaoning Technical University, 12(2), 213-216.

[7] Li, L. Liu, Z. \& Zhang, L. (2011). Prevalence and risk factors of bullying behaviors in college students (in Chinese). Chinese Jounary of School Health, 32(1), 57-58.

[8] Chapell, M., Casey, D., De la Cruz, C., Ferrell, J., Forman, J., Lipkin, R., Whittaker, S. (2004). Bullying in college by students and teachers. Adolescence, 39(153), 53-58.

[9] Chapell, M. S., Hasselman, S. L., Kitchin, T., Lomon, S. N., MacIver, K. W., \& Sarullo, P. L. (2006). Bullying in elementary school, high school, and college. Adolescence, 41(164), 633-648.

[10] Ottaway, S. A., Hayden, D. C., \& Oakes, M. A. (2001). Implicit attitudes and racism: Effects of word familiarity and frequency on the implicit association test. Social Cognition, 19, 97-144.

[11] Rothermund, K., \& Wentura, D. (2004). Underlying processes in the Implicit Association Test(IAT): Dissociating salience from associations. Journal of Experimental Psychology: General, 133, 139-165.

[12] Arkes, H. R., \& Tetlock, P. E. (2004). Attributions of Implicit Prejudice, or "Would Jesse Jackson 'Fail' the Implicit Association Test?" Psychological Inquiry, 15, 257-278.

[13] Messner, Claude; Vosgerau, Joachim (2010). Cognitive Inertia and the Implicit Association Test. Journal of Marketing Research (American Marketing Association), 47(2): 374-386.

[14] Grumm, M., Hein, S., \& Fingerle, M. (2011). Predicting aggressive behavior in children with the help of measures of implicit and explicit aggression. International Journal of Behavioral Development, 35(4), 352-357.

[15] Broerse, A., Crawford, T. J., \& den Boer, J. A. (2001). Parsing cognition in schizophrenia using saccadic eye movements: a selective overview. Neuropsychologia, 39(7), 742-756. 


\section{APPENDIX 1:}

The revised version of Olweus Bully/Victim Questionnaire

Introduction: It is aggressive behavior or intentional harm doing that is carried out repeatedly and over time in an interpersonal relationship characterized by an imbalance of power. It includes direct verbal attacks (saying mean and unpleasant things, or calling a student hurtful names), physical attacks (hitting, kicking, shoving), and more indirect psychological methods (such as deliberately excluding a student from a social group, spreading rumors).

Have you ever been bullied in your university by another student?

In the following there are 6 items, and the answer of each item by the frequency from A to $\mathrm{E}$.

Never B. Only once or twice C. Twice or three times a month D. Once a week

E. Several times a week

I have been called hurtful names by other students or roommates.

I have been excluded intentionally by other students or roommates.
I have been hitted, kicked, or shoved by other students or roommates.

Other students or roommates have spread rumors on me.

Other students have threaten me, or destroyed my belongings.

Other students have said mean and unpleasant things on me.

Have you ever bullied another student in your university?

In the following there are 6 items, and the answer of each item by the frequency from $A$ to $E$.

A. Never B. Only once or twice C. Twice or three times a month D. Once a week

E. Several times a week

7. I have called another student's hurtful names.

8. I have excluded intentionally another student or roommate from my social group.

9. I have hitted, kicked, and shoved another student.

10. I have spread another student's rumors.

11. I have threaten another student, or destroyed another student's belongings.

12. I have said mean and unpleasant things on another student. 